\title{
An Analytical Investigation of the Bullwhip Effect
}

\author{
Roger D. H. Warburton \\ University of Massachusetts, Dartmouth, North Dartmouth, Massachusetts 02747, USA \\ roger@warbs.net
}

\begin{abstract}
$\mathrm{T}$ he Bullwhip Effect is problematic: order variability increases as orders propagate along the supply chain. The fundamental differential delay equations for a retailer's inventory reacting to a surge in demand are solved exactly. Much of the rich and complex inventory behavior is determined by the replenishment delay. The analytical solutions agree with numerical integrations and previous control theory results. Managerially useful ordering strategies are proposed. Exact expressions are derived for the retailer's orders to the manufacturer, and the Bullwhip Effect arises naturally. The approach is quite general and applicable to a wide variety of supply chain problems.
\end{abstract}

Key words: Bullwhip Effect; supply chain management; ordering policy

Submissions and Acceptance: Received March 2002; revisions received November 2002 and May 2003; accepted October 2003 by Seungjin Wang.

\section{Introduction}

Lee, Padmanabhan, and Whang (1997a) and Lee, So, and Tang (2000) popularized the term "Bullwhip Effect," where a retailer's orders to their suppliers tend to have a larger variance than the consumer demand that triggered the orders. This demand distortion propagates upstream with amplification occurring at each echelon. Lee, Padmanabhan, and Whang (1997b) identified four major causes of the Bullwhip Effect: (1) users interpreting orders (the demand); (2) order batching; (3) promotions, which artificially stimulate demand; and (4) supply shortages, which also lead to artificial demands. The Bullwhip Effect has been documented as a significant problem in an experimental, managerial context (Sterman 1989), as well as in a wide variety of companies and industries (Buzzell, Quelch, and Salmon 1990; Kelly 1995; Holmstrom 1997; Metters 1997). Many proposed strategies for mitigating the Bullwhip Effect have a history of successful application (Clark 1994; Gill and Abend 1997; Hammond 1993; Towill 1997).

Fine (2000) discusses the Bullwhip Effect as one of two laws that govern supply chain dynamics, focusing on the strategic issues that arise. Anderson and Morrice (2000) analyzed the Bullwhip Effect in service industries, which cannot hold inventory, and in which backlogs can only be managed by adjusting capacity. Anderson, Fine, and
Parker (2000) suggest the amplification of demand volatility is particularly large in distribution and component parts supply chains, e.g., machine tools. Johnson and Whang (2002) survey emerging research on the impact of e-business on supply chains.

Much earlier, however, Forrester (1961) had defined a simplified form for the equations describing the relation between inventory and orders. In this paper, it is demonstrated that the fundamental differential delay equations describing an inventory reacting to a surge in consumer demand can be solved exactly. Forrester pioneered the simulation approach and established the importance of integrating information flow with material flow. Burbidge (1961) emphasized the now wellaccepted principles of cycle time reduction and order synchronization, and later coined his Law of Industrial Dynamics (Burbidge 1984): "If demand is transmitted along a series of inventories using stock control ordering, then the demand variation will increase with each transfer." Simulation has since been employed extensively to analyze supply chains (Berry and Towill 1995; Disney and Towill 2002a, 2002c).

\subsection{Related Theoretical Analyses}

Kahn (1987) showed that a serially correlated demand results in the Bullwhip Effect. Lee, Padmanabhan, and 
Whang (1997a) used the same demand assumption in which orders, $D_{t}$, depend on the orders in the previous time interval, $D_{t-1}$, as:

$$
D_{t}=\rho D_{t-1}+d+u_{t},
$$

where $d$ and $\rho$ are constants such that $d>0$ and -1 $<\rho<1$, and $u_{t}$ is normally distributed with zero mean and variance, $\sigma^{2}$. (Negative demands are unlikely when $\sigma \ll d$.) A cost minimization approach showed that distortion in demand arises when retailers optimize orders, and amplification increases as the replenishment lead-time increases. Various demand distributions and numerical experiments have been employed to study the Bullwhip Effect. Bourland, Powell, and Pyke (1996) examined the case in which the review period of the manufacturer is not synchronized with the retailer, while Gavirneni, Kapuscinski, and Tayur (1999) considered a manufacturer with limited capacity.

Disney and Towill (2002a) provide a useful compilation of the control theory literature applicable to the Bullwhip Effect. Dejonckheere, Disney, Lambrecht, and Towill (2002) used z-transforms to investigate bullwhip performance of order-up-to models. Particularly relevant is that John, Naim, and Towill (1994) used the Final Value Theorem to prove that, for step function shocks to the inventory, a long-term inventory deficit can occur; and they verified the prediction through simulation.

\section{The Retailer's Supply Chain}

Retailers attempt to minimize their inventory while maintaining sufficient on hand to guard against fluctuations in demand. The challenge is to formalize the ordering process with simple, robust policies that accomplish optimal inventory replenishment. The inventory, $I(t)$, is depleted by the demand rate, $D(t)$, and increased by the receiving rate, $R(t)$, so the inventory balance equation is:

$$
\frac{d I}{d t}=R(t)-D(t) .
$$

Initially $(t=0)$, the inventory has the value, $I_{o}$, which may be different from its desired value, $I_{D}$. We consider the impact of a step function surge in demand rate beginning at $t=0$, and assume the surge, $d$, to be constant and permanent. In Section 6, we discuss the impact of relaxing this assumption. The response to a deterministic step input is important because the responses are easily interpreted, and it is a useful measure of a system's ability to cope with sudden changes. The step function surge in demand is also a common feature in control theory analyses (John, Naim, and Towill 1994; Disney and Towill 2002a, 2002b).
As the consumer demand depletes the retailer's inventory, replenishment orders are issued to the manufacturer to bring the inventory back toward the desired value. A typical ordering policy is to order proportional to the inventory deficit:

$$
\begin{gathered}
O(t)=\frac{I_{D}-I(t)}{T} \text { for } I(t)<I_{D} \text { and } \\
O(t)=0 \quad \text { otherwise. }
\end{gathered}
$$

Forrester (1961) originally proposed the policy in (3a) and referred to the quantity, $T$, as the "adjustment time." This policy has been extensively studied, and both simulation and control theory analyses suggest it is misguided practice to attempt to recover the entire deficit in one time period, i.e., setting $T=1$ (Disney, Naim, and Towill 2000).

Equation (3b) adds the realistic constraint in which retailers and manufacturers stop ordering when their inventory exceeds its desired value. Using (3a) in that situation would represent negative production orders, i.e., returning items. Including (3b) is somewhat more realistic than previous approaches, all of which assume that excess inventory can be returned at no cost (Kahn 1987; Lee, Padmanabhan, and Whang 1997a, 1997b; Disney and Towill 2002c). We shall see that including (3b) significantly impacts inventory behavior. Due to manufacturing and shipping times, there is a delay in replenishment. The retailer's receipts from the manufacturer are the retailer's orders, just delayed by the replenishment time, $\tau$, which is assumed to be constant:

$$
R(t)=O(t-\tau) .
$$

If the manufacturer carries inventory, the replenishment time will be much shorter than if goods are made to order. However, in either case, the manufacturer, and not the retailer, determines the replenishment time. The adjustment time, $T$, allows the retailer to tune the order rate, and since $T$ can be adjusted more easily and quickly than $\tau$, it is reasonable to consider $\tau$ to be a constant.

The "inventory position" is usually considered to be the deficit minus the unfulfilled orders, or work in process (WIP), which was not included in the above equations. However, we present evidence in the Appendix that many of the critical features of the above equations should also occur in models that include WIP terms. For example, the inventory deficit predictions of this model are corroborated by results from control theory (John, Naim, and Towill 1994). Also, several of the model's theoretical implications (e.g., stability) depend directly on the replenishment delay, and any model or simulation that correctly treats the 
replenishment delay should inherit the stability properties discussed here. Therefore, even without the WIP term, the above ordering policy will turn out to be of considerable theoretical, as well as practical, interest.

Substituting the ordering policy in (2) and the time delay in (4) gives:

$$
\frac{d I}{d t}+\frac{I(t-\tau)}{T}=\frac{I_{D}}{T}-d .
$$

Because of the replenishment delay, no items are received for $t \leq \tau$, and (5) becomes:

$$
\frac{d I}{d t}=-d \Rightarrow I(t)=I_{o}-d t .
$$

Since the inventory is less than its desired value, the order rate, (3a), is for $t \leq \tau$ :

$$
O(t)=\left(I_{D}-I_{o}\right) / T+t d / T .
$$

The order rate has two contributions: a term attempting to fill the initial inventory deficit and another reacting to the demand.

\subsection{The Exact Solution}

The exact solution to (5) is derived in the Appendix. The critical feature is that the solution contains the Lambert $W$ function (Corless et al. 1996). The solution is for $t \leq \tau$ :

$$
I(t)=I_{o}-d t
$$

and for $t \geq \tau$ :

$$
\begin{array}{r}
I(t)=I_{D}-d T+A \exp [W t / \tau] \quad A=a+i \alpha \\
W=W(-\tau / T) \quad W=\omega+i \Omega \\
a=\left(e^{-\omega} / \Omega\right)[J(\Omega \cos \Omega+\omega \sin \Omega)-K \sin \Omega] \\
J=I_{o}-I_{D}+d(T-\tau) \\
\alpha=\left(e^{-\omega} / \Omega\right)[J(\omega \cos \Omega-\Omega \sin \Omega)-K \cos \Omega] \\
K=\left(I_{D}-I_{o}\right) \tau / \mathrm{T}-\mathrm{d} \tau .
\end{array}
$$

Equation (9) shows that the inventory behavior is dominated by the properties of the Lambert $W$ function, $W(z)$, which can be either real or complex, and whose real part can be either positive or negative (Corless et al. 1996). Figure 1 shows several solutions and verifies that the divergence of the inventory response is extremely sensitive to $\tau / T$, eloquently suggesting a relation to the Bullwhip Effect, which we will pursue in Section 4. The accuracy of these solutions is explored in the Appendix.

\subsection{Inventory Cycles}

We next demonstrate the importance of the real world constraint that the orders should go to zero when the inventory exceeds the desired value, (3b). The time at
Figure 1 Four exact solutions of the inventory equation. $T^{\star}$ is the critical adjustment rate, which brings the inventory exactly back to its desired value $(\tau=10)$.

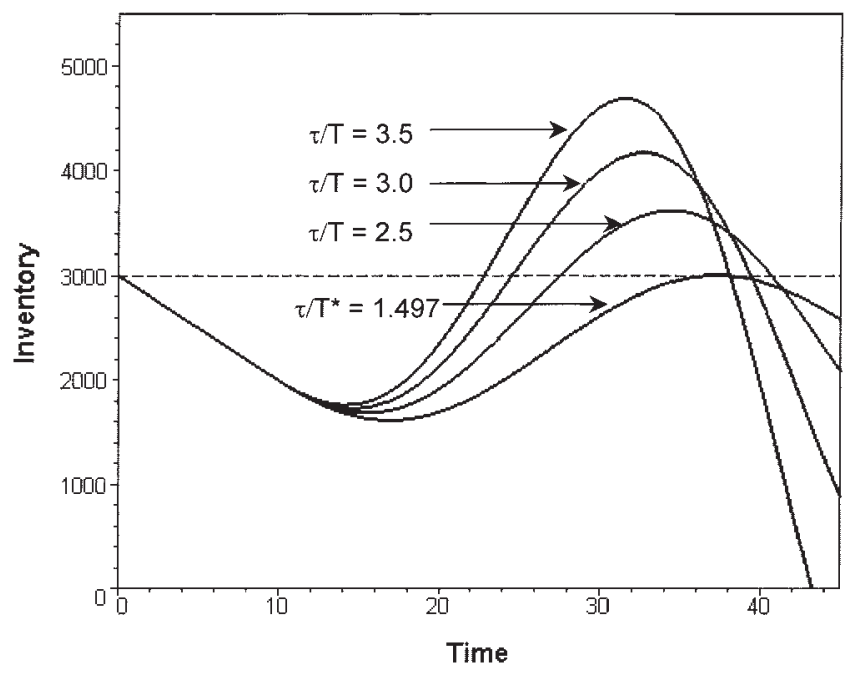

which the inventory returns to its desired value, $I_{D}$, is defined as $\mathrm{T}_{D}$. At $\mathrm{T}_{D}$, the order rate goes to zero, but items continue arriving, because there are still orders in the pipeline. The inventory overshoots and it takes a further time, $\tau$, the replenishment time, until the pipeline empties. After $t=T_{D}+\tau$, only the continuing consumer demand remains, and so (6) applies again. When the inventory falls to its desired level, the cycle repeats. The different characteristics of Figures 1 and 2 are due to the orders stopping when the inventory reaches its desired value.

\subsection{Permanent Inventory Deficits}

The theoretical solution in the stable regime predicts permanent inventory deficits. When $\tau / T<\pi / 2$, the real part of $W(z)$ is negative, and for large $t$, therefore, (9) becomes:

$$
I(t \rightarrow \infty) \rightarrow I_{D}-T d .
$$

The inventory falls to a value permanently below its desired value by $T d$. Using the Final Value Theorem, John, Naim, and Towill (1994) proved analytically that permanent deficits occur. Figure 3 shows solutions in this stable regime, which approach the predicted longterm deficits (dashed lines). For small $z>-1 / e, W(z)$ is real, and the solutions no longer oscillate, which can be seen in Figure 3, where the curve for $T=30$ shows no oscillation.

\section{Managing the Inventory}

Having derived the analytical solutions, we briefly explore some properties that will be useful when we discuss the Bullwhip Effect. The time of the inventory peak, referred to as $t^{*}$, can be calculated by setting 
Figure 2 The effect of the real world constraint in which the orders go to zero when the inventory reaches its desired value: $(3 b)(\tau$ $=10$ ).

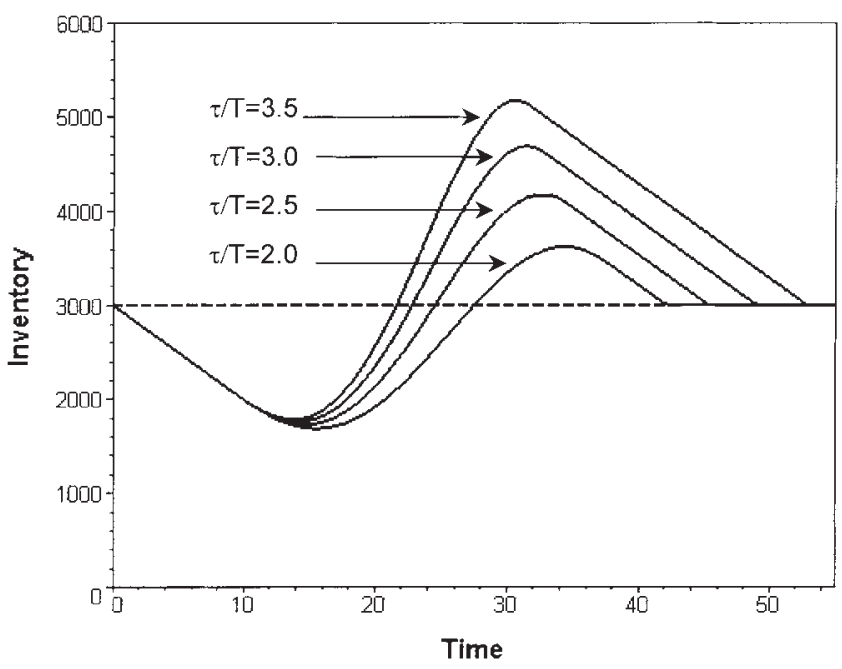

$d I / d t=0$ in (9). Since $A$ and $W$ are complex, one obtains:

$\frac{A W}{\tau} \exp \left\{W t^{*} / \tau\right\}=0 \Rightarrow t^{*}=\frac{\tau}{\Omega} \tan ^{-1}\left[\frac{a \omega-\alpha \Omega}{\alpha \omega+a \Omega}\right]$.

When a retailer detects a surge in consumer demand, the time of the peak in inventory can be forecast. The size of the peak will depend on the accuracy of the estimate of $d$, but the analytical solutions make the computations quite straightforward.

Figure 2 showed that, for long replenishment delays, the inventory experiences a significant (and problematic) overshoot, while Figure 3 shows that inventory deficits occur for small values of $\tau / T$. Therefore, there is a critical value of the adjustment time, $T^{*}$, for which the inventory climbs back to its desired value without any overshoot, i.e., $I\left(t^{*}\right)=I_{D}$ :

$$
\left.I_{D}=I_{D}-d T^{*}+A \exp \left(W t^{*} / \tau\right)\right) .
$$

Since the argument of $W\left(-\tau / T^{*}\right)$ depends on $T^{*}$, the above equation must be solved numerically. Figure 1 includes the inventory response for the critical value of the adjustment time, $T^{*}$. This provides another potentially valuable tool. While the manufacturer determines the delay time, the parameter, $T$, was introduced to allow the retailer to control the inventory response. If a surge in demand is detected and estimated, the retailer can adjust the orders (i.e., select $T$ $=T^{*}$ ) so that the inventory is made up without suffering either deficits or overshoots.

\section{The Bullwhip Effect}

The Bullwhip Effect is defined as the amplification of order variability along the supply chain. The retailer's orders are given by $O(t)=\left(I_{D}-I(t)\right) / T$, and we now have the exact solution for the inventory in (9). Figure 4 shows a plot of the retailer's order rate, which quickly grows to exceed the constant consumer demand rate. The orders go to zero when the inventory exceeds its desired value, and remain so for the rest of the inventory cycle. The impact of the zero order rate is noteworthy, as it affects a significant fraction of the cycle. (Figure 4 also includes the manufacturer's initial order rate to the supplier, which is discussed in Section 5.)

One measure of the Bullwhip Effect is the ratio of the output order rate (retailer orders to manufacturer) to the input order rate (consumer demand). The retail order rate climbs to a peak, which occurs soon after $\tau$,

Figure 3 Stable solutions with the prediction of long-term, permanent inventory deficits (dashed lines).

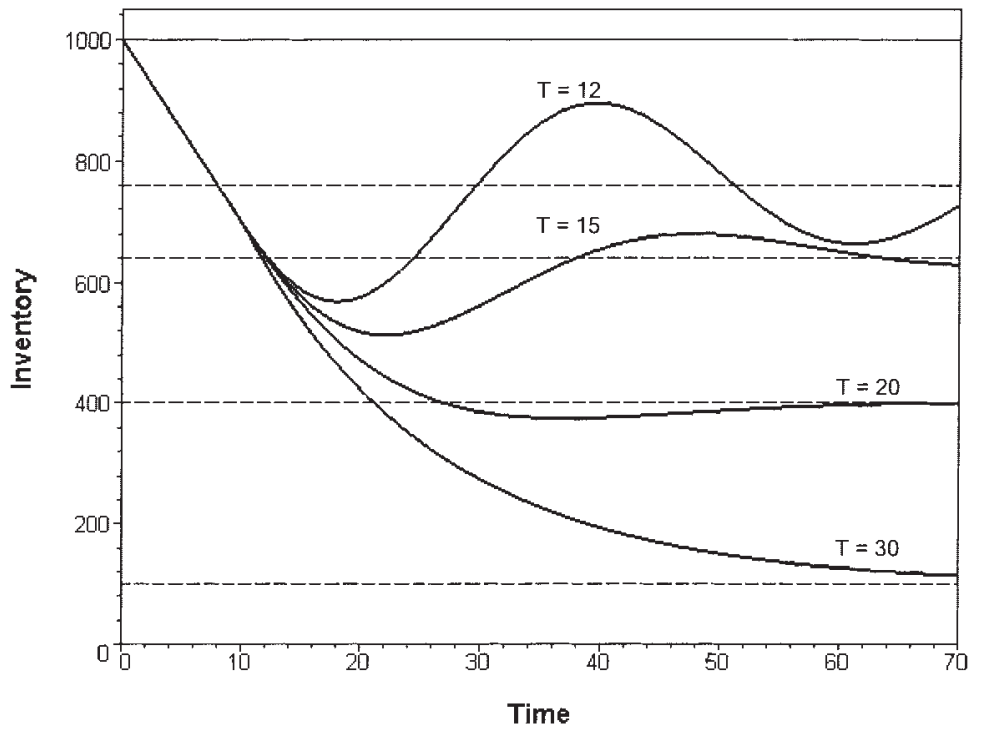


Figure 4 The "Bullwhip Effect": the amplification of order rate from consumer to retailer to manufacturer. The flood and drought in retail orders are also apparent.

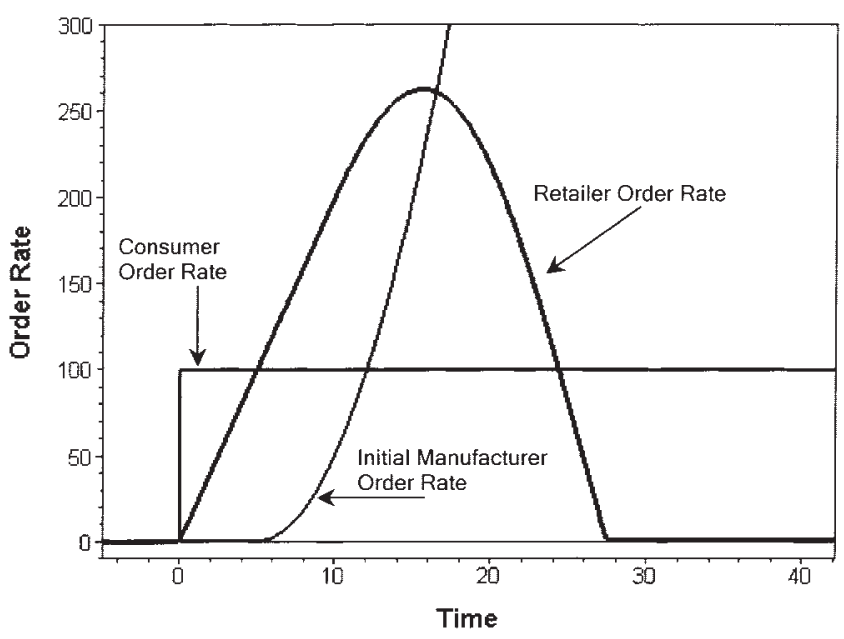

and so this is an appropriate time at which to compare the rates:

$$
B W^{R}=\frac{O(\tau)}{d}=\frac{I_{D}-I(\tau)}{T d}=\frac{\tau}{T} .
$$

The superscript, $R$, indicates that this amplification in orders is attributable to the retailer's ordering policy. The Bullwhip Effect is usually defined in terms of the instantaneous variability in orders, and (15) reflects this in its use of order rates. Since we have analytical solutions, the relative contributions of the parameters are explicit. For example, (15) is independent of the size of the surge, which is a direct consequence of defining the Bullwhip Effect as a ratio. Equation (15) also suggests that increasing the value of $T$ can reduce the Bullwhip Effect. (We continue to assume that the replenishment delay is not easily changeable by the retailer.) However, from Section 2, we know that $T$ cannot be raised arbitrarily without permanent inventory deficits occurring. If it is important for the inventory to return to its desired value, then some Bullwhip Effect is inevitable, e.g., $T^{*}$ is the logical choice because it returns the inventory to its desired value without overshoot, and for $\tau=10, T^{*}$ $=6.68$, and $B W^{R}=1.5$.

The definition in (15) only represents the orders in the early stages of the inventory cycle, and does not account for the inventory overshoot. Figure 4 shows the retailer's orders as a flood followed by a drought, and the longer the delay time, the greater the overshoot, and the longer the drought. This suggests a different calibration of the Bullwhip Effect: measuring the growth in inventory fluctuations, rather than order fluctuations. For example, in Figure 2, one can compare the peak in the inventory (overshoot) to the bottom of the decline (undershoot). The undershoot is defined as the decline through the replenishment time, during which the inventory falls by the amount $d \tau$. Therefore,

$$
B W_{I}^{R}=\frac{\text { overshoot }}{\text { undershoot }}=\frac{I^{*}-I_{D}}{d \tau} .
$$

This measure of the Bullwhip Effect has a different character from that in (15), because the behavior of the overshoot is different from that of the orders. For example, in the critical $T^{*}$ case, the overshoot was adjusted to be zero, $\left(I^{*}=I_{D}\right)$ and so $B W_{I}^{R}=0$. In other words, if the Bullwhip in inventory is adjusted to zero, the Bullwhip in orders may remain. If it is more important to replenish the inventory than to minimize order fluctuations, then (16) is a better measure of the impact of demand fluctuations. Bullwhip analyses typically concentrate on order variability, and while there is clearly a cost benefit to its reduction, other impacts, such as inventory replenishment, should be considered. For example, customer service levels will depend on the ability to replenish the inventory. Fransoo and Wouters (2000) cautioned that there are many ways to measure the Bullwhip Effect, an argument bolstered by the above discussion. The manufacturer will add yet another contribution to the Bullwhip Effect, which is discussed below.

\section{The Manufacturer's View}

We now discuss the impact on the manufacturer of the orders from the retailer. The equation for the change in the manufacturer's inventory is similar to that of the retailer:

$$
d I^{M} / d t=R^{M}(t)-S^{M}(t) .
$$

Since both retail and manufacturing quantities occur in the equations, the superscript, $M$, is included to distinguish the manufacturer's quantities. $R^{M}$ represents the receiving rate, which can be either from an upstream supplier, or from the manufacturer's own, internal production. The decline, $S^{M}$, represents the shipment rate to the retailer. We assume that, before the retailer starts ordering, the manufacturer's inventory is at the desired value.

The receiving rate from the upstream supplier will depend on the orders issued by the manufacturer and the associated replenishment delay. The same process applies if the items are produced internally; the manufacturer is then issuing internal orders. The manufacturer's situation is frequently more complicated than the retailer's because orders to suppliers often represent sub-component orders rather than complete items. Typically, the sub-components have different manufacturing and shipment delays. Additionally, manufacturers usually supply several retailers. However, the general approach used in the retail case still 
applies: The manufacturer creates an ordering policy for each item, and the receipts from the supplier will be characterized by the supplier's replenishment delay time for that item.

Before the supplier has fulfilled any orders, the receipts, $R^{M}$, will be zero. Therefore, initially, we can ignore the upstream supplier and consider just the interaction between the manufacturer and the retailer. The manufacturer's inventory declines as items are shipped to the retailer, and these shipments are equal to the orders received from the retailer. There will be a delay in shipping, which we refer to as the manufacturing time, $\tau^{M}$. When shipments begin, they reflect the orders received at time $t-\tau^{M}$ :

$$
S^{M}(t)=O^{R}\left(t-\tau^{M}\right) .
$$

The manufacturer faces the same dilemma as the retailer: What ordering policy to employ? We again propose Forrester's order rate, and so the manufacturer's orders are:

$$
\begin{gathered}
O^{M}(t)=\frac{I_{D}^{M}-I^{M}(t)}{T^{M}} \text { for } I^{M}(t)<I_{D}^{M} \text {, and } \\
O^{M}(t)=0 \text { otherwise. }
\end{gathered}
$$

The retailer's initial orders are given by (7). Assuming the retailer's inventory starts at the desired value $\left(I_{D}\right.$ $\left.=I_{O}\right)$, and including the manufacturer's time delay, one obtains the equation for the manufacturer's inventory as:

$$
d I^{M} / d t=-S^{M}(t)=-O^{R}\left(t-\tau^{M}\right)=-d\left(t-\tau^{M}\right) / T,
$$

which has the solution:

$$
I(t)=I_{o}^{M}-d\left(t-\tau^{M}\right)^{2} / 2 T .
$$

This solution is valid until the shipments begin arriving from the supplier. We will not discuss that case, but the exact retailer order expressions could be used and the integrals performed. Equation (21) describes the initial response of the manufacturer's inventory, and this is also shown in Figure 4. It can be seen that, after a delay, the manufacturer's order rate rises, and even faster than the retailer's.

\subsection{Manufacturer's Contribution to the Bullwhip Effect}

Equation (21) shows that the impact of the retailer's orders is to cause the manufacturer's inventory to decline as a quadratic function of time. This is in contrast to the linear decline in the retailer's inventory, (6). Using (21) for the manufacturer's inventory, the manufacturer's order rate to the supplier is:

$$
O^{M}(t)=\frac{d\left(t-\tau^{M}\right)^{2}}{T^{M} T} .
$$

The manufacturer's order rate to the supplier initially grows as a quadratic function of time. This is in stark contrast to the retailer's order rate, which initially grows linearly, and the constant consumer demand rate, which started the whole order train (see Figure 4). The Bullwhip Effect again emerges. Further, the manufacturer does not realize that he is witnessing the flood part of the order cycle from the retailer, and that a drought is to follow.

The Bullwhip Effect caused by the manufacturer can be measured as the ratio of supplier order rate (output by manufacturer) to retail order rate (input to manufacturer). Using the fact that the orders peak close to when the supplier items begin arriving, $t=\tau^{S}+\tau^{M}$ :

$$
B W^{M}=\frac{\left(\tau^{S}\right)^{2}}{2 \tau T^{M}} .
$$

Equation (23) measures the amplification in orders solely attributable to the manufacturer. However, the manufacturer's magnification of the order rate compounds that of the retailer's. The input orders to the manufacturer are the orders output from the retailer, which are an amplified version of the consumer demand. Therefore, the total Bullwhip Effect from consumer to supplier is the combination

$$
B W^{C \rightarrow S}=\frac{d\left(\tau^{S}\right)^{2} / 2 T T^{M}}{d}=B W^{R} B W^{M} .
$$

Equation (24) shows the multiplicative nature of the Bullwhip Effect (Dejonckheere, Disney, Lambrecht, and Towill 2002).

\section{Discussion}

\subsection{Historical Remark}

We begin by briefly discussing why the results presented here differ from Forrester's classic treatment (1961). Forrester defined a pool of goods on order, $G(t)$, which increases as orders are issued, and is depleted by items going into inventory. Forrester approximated the fulfillment delay by assuming that the inventory receiving rate is a constant fraction $\left(1 / T_{G}\right)$ of the accumulated goods on order, i.e., items flow into inventory proportional to the backlog. $T_{G}$ is referred to as the "exponential delay," and is intended to play a role similar to $\tau$ in the delay case. However, under Forrester's assumption, ordering more items increases the backlog and immediately results in increased deliveries. In the delay case, orders are delivered after the replenishment delay, and in the same sequence as they are ordered. The equations are:

$$
\frac{d G(t)}{d t}=O(t)-R(t) \quad R(t)=G(t) / T_{G}
$$


The order rate is again given by Forrester's expression, (3a), which results in an ordinary, second order differential equation for the inventory:

$$
I^{\prime \prime}+I^{\prime} / T_{G}+I^{\prime} / T_{G} T=I_{D} / T_{G} T .
$$

Forrester ignored the real world constraint in which the order rate goes to zero when the inventory exceeds its desired value. The solution to (26) is:

$$
I=I_{D}+\exp (\alpha t)[A \sin (\beta t)+B \cos (\beta t)] .
$$

Substitution into the homogeneous equation determines the constants $\alpha$ and $\beta$, while the boundary conditions $\left(G(0)=G o\right.$ and $\left.d I / d t=G o / T_{G}\right)$ determine the constants $A$ and $B$ :

$$
\begin{aligned}
\alpha=-1 /\left(2 T_{G}\right) \quad \beta^{2}=\frac{1}{T_{G} T}\left(1-\frac{T}{4 T_{G}}\right) & \\
A & =\frac{G_{o}}{\beta T_{G}}-\frac{\left(I_{o}-I_{D}\right) \alpha}{\beta} \quad B=I_{o}-I_{D} .
\end{aligned}
$$

In the above solution, the exponential term, $\alpha$, is always negative, guaranteeing stable solutions in which the inventory always approaches the desired value, and which Forrester referred to as "second order negative feedback." The significant difference from the solution to the delay equation is that, while the constant $\alpha$ is always negative in Forrester's case, $\omega$ can be both positive and negative in the delay equation case. Forrester's solutions are always stable, but the delay equation includes both stable and unstable regimes. Figure 5 shows the exact solution to Forrester's approximate equation for values that he typically used: $T_{G}=10$ and $T=5$.

Figure 5 also shows the delay equation solution for the same case (the demand, $d=0$, and $\tau=10, T=5$ ).
In distinct contrast to the Forrester case, the inventory diverges. It is moderately interesting that Forrester chose parameter values that are stable, but which generate unstable solutions when the more realistic replenishment delays are considered.

\subsection{Finite Time Length Demand}

We now consider a surge in demand that lasts for a finite time, $T_{S}$, and then falls to zero. The solution in (9) is still valid up through $T_{\mathrm{S}}$. After $T_{\mathrm{S}}$, the demand is zero, but there are still items in the pipeline, and these continue arriving until $t=T_{\mathrm{S}}+\tau$. If at this point the inventory exceeds its desired value, the order rate will remain zero. Since there are no outstanding orders and no demand, the inventory is constant. Some examples of the inventory behavior when the demand goes to zero are shown in Figure 6. While the length of the surge is finite, and its length affects the shape and scale of the response, the end result is frequently an expensive overshoot of the inventory. The interesting inventory behavior is again determined by the Lambert $W$ function, which is therefore crucial in understanding the details of the response.

\section{Conclusions}

We have demonstrated that it is possible to solve exactly the delay differential equation describing the inventory. No approximations were required. The replenishment delay emerges as responsible for much of the rich and complex behavior associated with the inventory response. The ordering policy is rather more general than previous theoretical analyses because it includes the realistic condition that the order rate is zero once the inventory reaches its desired value.

While the constant demand case was considered, it

Figure 5 Comparison of stable Forrester and unstable Lambert $W$-based solutions.

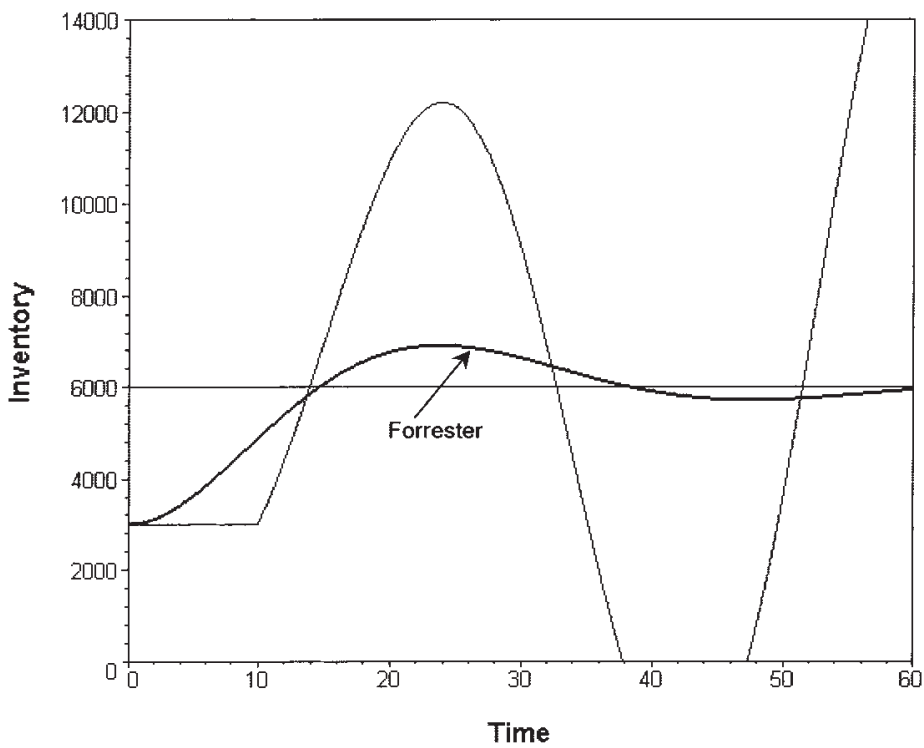


Figure 6 The inventory response for surges in demand that last for a finite time, $T_{s}(\tau=10)$.

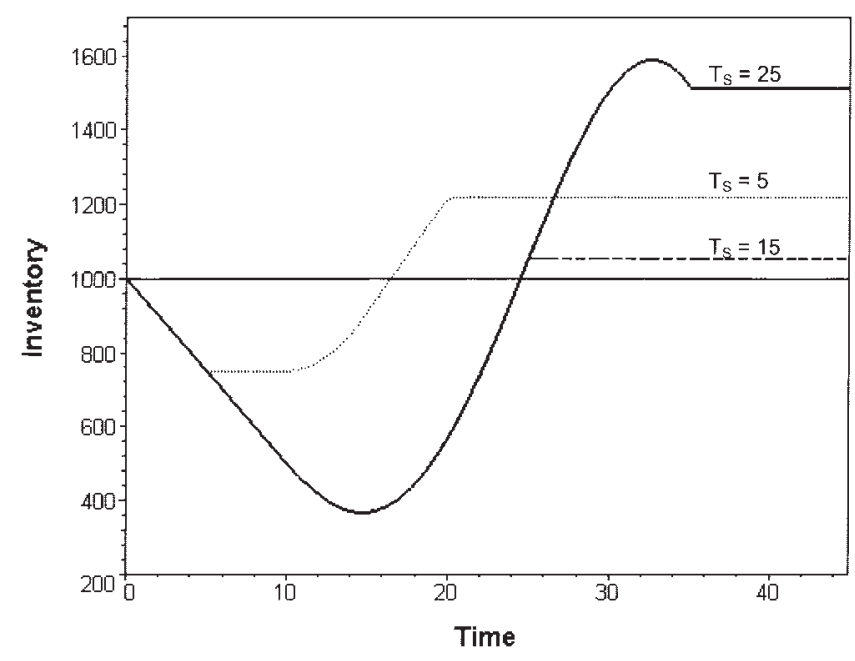

is not true that the approach only applies to this assumption. The demand term only contributes to the inhomogeneous equation, and a different form for the demand would result in a new inhomogeneous solution, but the homogeneous Lambert $W$-based solution would remain unchanged. Thus, the process is completely general, and applicable to any form for the demand.

More important, however, is that, for any demand for which the inhomogeneous solution can be found, the full solution will always contain the same homogeneous solution and, therefore, inherit its properties (i.e., the Lambert $W$ function). This was confirmed in Figure 6, where even though the demand was turned off, the inventory response was still dominated by the Lambert $W$ function. Also, this model's prediction of permanent inventory deficits reproduces behavior found in more complex models previously analyzed with control theory.

Comparison with direct numerical integration (see Appendix) established that the theoretical solutions provide an excellent representation of the inventory behavior in both the stable and unstable regimes. The theory predicts a critical stability point, which is of considerable practical value, since a diverging inventory is expensive. Numerical integrations verified that the critical stability point is an inherent feature of the differential equation, and its value is accurately predicted by the theory. The ordering policy was parameterized so as to allow the retailer to calculate a critical order adjustment rate that returns the inventory back to its desired value exponentially fast, while not generating an overshoot. The availability of analytical solutions makes such computations quite straightforward. Confidence is thus established in both the accuracy and applicability of the Lambert $W$ functionbased analytical solutions.
We calculated the replenishment rate for orders issued by the retailer to the manufacturer, and solved the equation for the manufacturer's inventory reacting to the retailer's orders. The Bullwhip Effect emerged naturally. The manufacturer's situation is complicated by shipments to multiple retailers and orders to many suppliers. In principle, however, the manufacturer can also create a critical ordering policy to reduce inventory overshoot, but must also communicate the size of the consumer demand to the supplier. This reinforces the suggestion that the sharing of retail sales information is a major strategy for countering the Bullwhip Effect (Lee, Padmanabhan, and Whang 2000; Towill 1996). The additional Bullwhip Effect attributable to the manufacturer was calculated.

Bullwhip analyses are typically based on inventory cost minimization, or profit maximization (Kahn 1987; Lee, Padmanabhan, and Whang 1997b). Here, we took a rather different approach. A form for the consumer demand was proposed, and solving the resulting equations determined the inventory behavior over time. It was then possible to adjust the parameters to achieve a desired inventory response. It emerged that one must trade the reduction in the Bullwhip Effect against competing processes, such as permanent inventory deficits or inventory excesses.

Using simulation, it is difficult to understand a system's underlying structure and behavior, and so opportunities for performance enhancements may be missed (Towill 1989). The model described here can help determine the interesting regions in the parameter state space, and aid direct experimentation with simulation. We conclude that these analytical case studies are extremely useful in precisely defining and quantifying the inventory behavior, and they provide tools to analyze important management issues, such as the Bullwhip Effect. The solutions clarify the contribution of the various parameters, which helps in assessing and measuring the relative impact of proposed remedies. All of the presented solutions are exact and deterministic. Somewhat surprisingly, the interesting scenarios are mathematically quite tractable, and so the general approach introduced here should prove useful in a wide variety of supply chain problems.

\section{Acknowledgments}

This work was supported in part by Griffin Manufacturing Co., Inc. and a grant from the National Textile Center. Steven Warner and Jonathan Hodgson contributed excellent suggestions. The author would like to thank the referees, whose questions resulted in new insights.

\section{Appendix}

\section{A.1. The Exact Solution}

In this section, we derive the exact solution to (5) for $t>\tau$. As is usual, we begin with the homogeneous equation: 


$$
\frac{d I}{d t}+\frac{I(t-\tau)}{T}=0
$$

The complexity of this equation is associated with the replenishment delay. The equation is referred to as the logistics equation, and is a member of the class of delay differential equations (Bellman and Cooke 1963). We propose a solution of the form $I=A \exp (\mathrm{st})$, to obtain:

$$
A e^{s t}\left\{s+e^{-s \tau} / T\right\}=0 .
$$

The term in braces can be rearranged into the following suggestive form:

$$
s \tau e^{s \tau}=-\tau / T .
$$

Equation (A.3) can be solved exactly in terms of the Lambert $W$ function, which is defined as:

$$
W(z) e^{W(z)}=z .
$$

A review of the history, theory, and applications of the Lambert $W$ function may be found in Corless et al. (1996). There are an infinite number of complex values of the Lambert $W$ function, denoted as $W(k, z)$. By the linearity of (A.1), any combination of the $W(k, z)$ can be used in the solution, and we could consider a solution of the form:

$$
I(t)=\sum_{k} c_{k} \exp [W(k,-\tau / T) t / \tau] .
$$

This is an infinite formula and we are interested in practical, easy-to-implement solutions. It turns out that using just the first term $(k=0)$ results in a sufficiently accurate representation of the inventory response. Therefore, our "practical" solution is:

$$
I(t)=A \exp (W t / \tau) \quad \text { with } \quad W=W(0,-\tau / T) .
$$

The inventory behavior is extremely sensitive to the properties of $W(z)$, because it occurs in the exponential. Fortunately, the Lambert $W$ function is readily available in efficient and accurate implementations, such as in MAPLE (2002), where it is defined as Lambert $W(k, z) . W(z)$ only enters (A.6) as a parameter; there is no time dependence in the $W(z)$ term.

We now proceed to solve the inhomogeneous equation. The inhomogeneous term is a constant, so we propose the constant, $K$, as a solution. Substitution in (5) determines $K$ as $K=I_{D}-d T$. Adding this to the homogeneous solution gives:

$$
I(t)=I_{D}-d T+A \exp [W t / \tau] .
$$

The constant, $A$, is determined by the imposition of boundary conditions. The inventory must be continuous, and so (A.7) must match the solution in (6) at $t=\tau$. In order to guarantee appropriate behavior at $t=\tau$, it is also necessary to specify the slope of the inventory there, and (2) provides the required condition. Two conditions on the inventory require two integration constants. Noting that $W$ is complex suggests that $A$ can be also. Treating $A$ as a complex constant results in a solution that turns out to provide an excellent representation of the inventory over the entire range of $\tau / T$. Therefore, we consider $A$ and $W$ as:

$$
A=a+i \alpha \quad W=\omega+i \Omega .
$$

Matching the solution in (6) to that of (A.7) at $t=\tau$, gives:

$$
a \cos \Omega-\alpha \sin \Omega=e^{-\omega}\left(I_{o}-I_{D}+d(T-\tau)\right) .
$$

Differentiating (A.7) at $t=\tau$, gives the required condition on the derivative:

$$
\begin{aligned}
\left.\frac{d I}{d t}\right|_{t=\tau}=R(\tau)-d=O(0)- & \\
& =\frac{I_{D}-I_{o}}{T}-d=\frac{A W}{\tau} e^{W} .
\end{aligned}
$$

This provides a second relation between $a$ and $\alpha$, which can thus be determined in terms of $\omega$ and $\Omega$. The complete, exact solution to (5) is thus determined, and is given in (9).

A number of combinations of solutions from (A.5) were tried. Despite adding complexity, including more Lambert $W$ functions, doesn't significantly improve the match to the numerical solution. Considering the constant, $A$, as complex, provides two parameters, and guarantees the continuity of the solution and its derivative at $t=\tau$. From a practical perspective, once the inventory climbs back to $I_{D}$, (3b) applies, and the solution changes. In practice, therefore, the Lambert $W$ function is only required until $I=I_{D}$. We conclude that the $k=0$ term with a complex constant provides a practical representation of the inventory behavior in both the stable and unstable regimes.

\section{A.2. The Accuracy of the Theoretical Solution}

The above discussion suggests that only the first term in the series in (A.5) is required, and we now examine the accuracy of that assumption. Figure 7 compares the analytical solutions with numerical integrations of the same differential equation. The difference between the theoretical and numerical solutions in the unstable regime is less than 3\% at the first peak. In the stable regime, there is very little difference between the theoretical and numerical solutions. The theoretical solution also predicts the period of oscillation to better than $0.4 \%$. Therefore, in practical situations where there is likely to be noise in the data, the one-term Lambert $W$ function provides an easy-to-compute, accurate representation of the inventory response.

\section{A.3. The Impact of the Lambert $W$ Function}

The properties of the solutions are determined by the characteristics of the Lambert $W$ function. For large $\tau / T$, the real part of the Lambert function, $\omega$, is positive and the solutions diverge (Figure 1). However, for small values of $\tau / T=\epsilon$, $W(-\epsilon)=-\epsilon$, which is real, and results in stable, decaying solutions. The issue is to determine the critical stability point. There are two interesting values for the argument of $W(z),-1 / e$ and $-\pi / 2$.

When $\tau / T>\pi / 2$, the real part of $W(z)$ is positive, and the solution in (9) applies. The oscillation is due to the imaginary part of the Lambert function, $\Omega$. As $\tau / T$ increases, $\Omega$ increases, resulting in a decrease in the period, which can be clearly seen in Figure 1.

The real part of $W(z)=0$ at $-\pi / 2$. Therefore, the transition from unstable to stable solutions occurs when $\tau / T=\pi / 2$. A detailed numerical integration of the delay equation was 
Figure 7 Comparison of theoretical and numerical solutions, showing differences of less than $3 \%$ (at the peak) in the unstable regime, and small differences in the stable regime $(\tau=10)$.

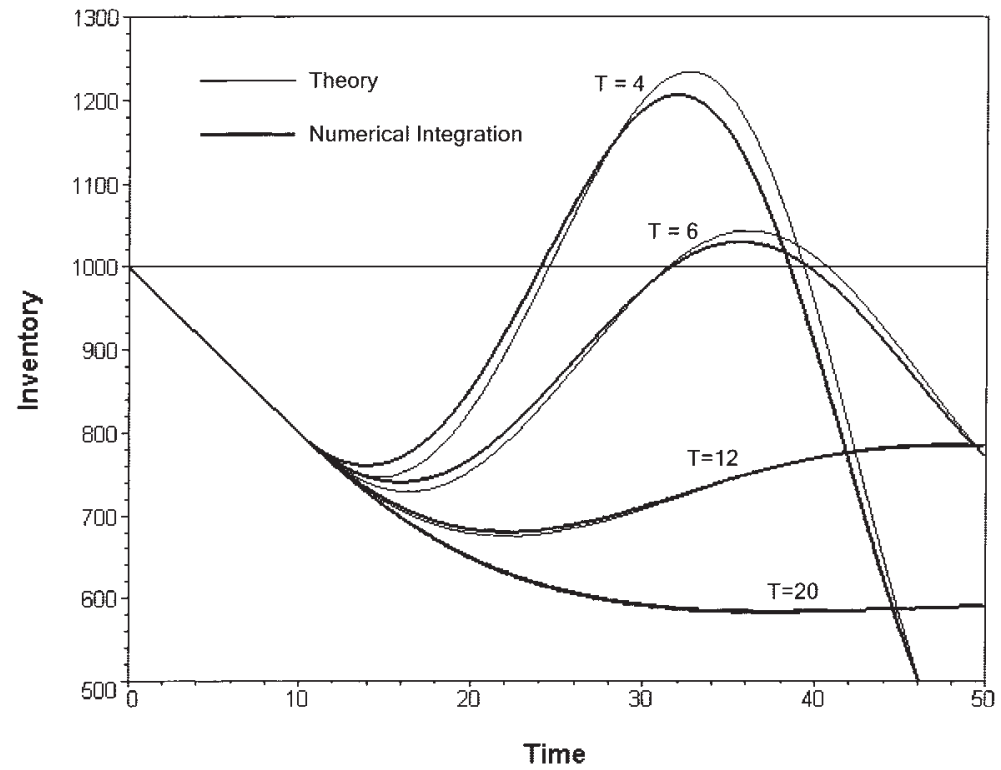

performed on either side of the critical stability value $(\tau$ $=10.0 \Rightarrow T=6.36 \pm 0.1)$. One slowly diverged and the other slowly converged, validating that the critical stability point is in fact an inherent feature of the differential equation. The ability to predict this critical stability point is very valuable, because it divides stable from unstable inventory behavior.

\section{A.4. Analysis of Assumptions}

While we considered the constant demand case, it is not true that the solution only applies to this assumption. Since (5) is linear, its solution is always the sum of homogeneous and inhomogeneous components. A different demand would result in a new inhomogeneous component, but the homogeneous Lambert $W$-based component would remain unchanged. Therefore, the process is completely general and applicable to any form for the demand.

The analytical solutions reproduced the inventory deficit prediction of control theory, and we can hypothesize as to why the simplified model (without a WIP term) includes such behavior found in more complex models. The critical stability point and other interesting characteristics are properties of the exponential Lambert $W$ function, which arises from the solution to the homogeneous equation. Therefore, any system of equations that includes the replenishment delay (a homogeneous term) should inherit these characteristics. In contrast, the demand leads to inhomogeneous terms, e.g., $d$ is on the right-hand side of (5). As long as the ordering policy includes a time delay in fulfillment, adding WIP terms should merely add inhomogeneous terms to the homogeneous Lambert $W$ solutions. We tentatively conclude that it is reasonable to expect more complex models to inherit the properties described here. In which case, despite its apparent simplicity, the present model has significant predictive power, and is of considerable theoretical interest. Further research is underway to confirm this conjecture.

\section{References}

Anderson Jr., E. G., D. J. Morrice. 2000. A simulation game for service-oriented supply chain management: Does information sharing help managers with service capacity decisions? Production and Operations Management 9(1) 40-55.

Anderson Jr., E. G., C. H. Fine, G. G. Parker. 2000. Upstream volatility in the supply chain: The machine tool industry as a case study. Production and Operations Management 9(3) 239-261.

Bellman, R. E., K. L. Cooke. 1963. Differential-difference equations. Academic Press, New York.

Berry, D., D. R. Towill. 1995. Reduce costs: Use a more intelligent production and inventory policy. BPICS Control Journal 1(7) $26-30$.

Bourland, K., S. Powell, D. Pyke. 1996. Exploring timely demand information to reduce inventories. European Journal of Operations Research 92 239-253.

Burbidge, J. L. 1961. The "new approach" to production. Production Engineer 40 3-19.

Burbidge, J. L. 1984. Automated production control with a simulation capability. Proceedings of IFIP Conference WG 5-7, Copenhagen, $1-14$.

Buzzell, R. D., J. A. Quelch, W. J. Salmon. 1990. The costly bargain of trade promotion. Harvard Business Review 68(March April) 141-148.

Clark, T. 1994. Campbell Soup: A leader in continuous replenishment innovations. Harvard Business School Case, Boston, Massachusetts.

Corless, R. M., G. H. Gonnet, D. E. G. Hare, D. J. Jeffrey, D. E. Knuth. 1996. On the Lambert $\mathrm{W}$ function. Advances in Computational Mathematics 5 329-359.

Dejonckheere, J., S. M. Disney, M. R. Lambrecht, D. R. Towill. 2002. Transfer function analysis of forecasting induced bullwhip in supply chains. International Journal of Production Economics $\mathbf{7 8}$ 133-144.

Disney, S. M., M. M. Naim, D. R. Towill. 2000. Genetic algorithm optimization of a class of inventory control systems. International Journal of Production Economics 68 259-278.

Disney, S. M., D. R. Towill. 2002a. Inventory drift and instability in 
Warburton: An Analytical Investigation of the Bullwhip Effect Production and Operations Management 13(2), pp. 150-160, () 2004 Production and Operations Management Society

order-up-to replenishment policies. Proceedings of the 12th International Symposium on Inventories, August, Budapest, Hungary.

Disney, S. M., D. R. Towill. 2002b. A discrete transfer function model to determine the dynamic stability of a vendor managed inventory supply chain. International Journal of Production Research 40(1) 179-204.

Disney, S. M., D. R. Towill. 2002c. A procedure for the optimization of the dynamic response of a vendor managed inventory system. Computers and Industrial Engineering $4327-58$.

Fine, C. 2000. Clockspeed-based strategies for supply chain design. Production and Operations Management 9(3) 213-221.

Forrester, J. W., 1961. Industrial Dynamics, MIT Press, Cambridge, Massachusetts.

Fransoo, M., J. F. Wouters. 2000. Measuring the bullwhip effect in the supply chain. Supply Chain Management 5(2) 78.

Gavirneni, S., R. Kapuscinski, S. Tayur. 1999. Value of information in capacitated supply chains. Management Science 45(1) 16-24.

Gill, P., J. Abend. 1997. Wal-Mart: The supply chain heavyweight champ. Supply Chain Management Review 1(1) 8-16.

Hammond, J. 1993. Quick response in retail/manufacturing channels in Globalization, technology and competition: The fusion of computers and telecommunication in the 1990's, Bradley et al. (ed.), Harvard Business School Press, Boston, Massachusetts, 185214.

Holmstrom, J. 1997. Product range management: a case study of supply chain operations in the European grocery industry. Supply Chain Management 2(3) 107-115.

John, S., M. M. Naim, D. R. Towill. 1994. Dynamic analysis of a WIP compensated decision support system. International Journal of Manufacturing Design 1(4) 283-297.
Johnson, M. E., S. Whang. 2002. E-business and supply chain management: An overview and framework. Production and Operations Management 11(4) 413-423.

Kahn, J. 1987. Inventories and the volatility of production. American Economic Review 77 667-679.

Kelly, K. 1995. Burned by busy signals: Why Motorola ramped up production way past demand. Business Week 636.

Lee, H. L., V. Padmanabhan, S. Whang. 1997a. Information distortion in the supply chain: The Bullwhip Effect. Management Science 43(4) 546-558.

Lee, H. L., V. Padmanabhan, S. Whang. 1997b. The bullwhip effect in supply chains. Sloan Management Review 38(3) 93-102.

Lee, H. L., K. C. So, C. S. Tang. 2000. The value of information sharing in a two-level supply chain. Management Science 46(5) 626-643.

MAPLE. 2002. Computer Program, Version 8. Waterloo Maple, Inc., Ontario, Canada.

Metters, R. 1997. Quantifying the bullwhip effect in supply chains. Journal of Operations Management, Columbia, May.

Sterman, J. D. 1989. Modeling managerial behavior: Misperceptions of feedback in a dynamic decision-making experiment. Management Science 35(3) 321-339.

Towill, D. 1989. The dynamic analysis approach to manufacturing systems design. Journal of Advanced Management Engineering $\mathbf{1}$ 131-140.

Towill, D. 1996. Time compression and the supply chain: A guided tour. Supply Chain Management 1(1) 15-27.

Towill, D. 1997. FORRIDGE: Principles of good practice in material flow. Production Planning and Control 8(7) 622-632. 